\title{
Long-range memory and non-Markov statistical effects in human sensorimotor coordination
}

\author{
Renat M. Yulmetyev a,*, Natalya Emelyanova ${ }^{\mathrm{a}}$, Peter Hänggi ${ }^{\mathrm{b}}$, \\ Fail Gafarov ${ }^{\mathrm{a}}$, Alexander Prokhorov ${ }^{\mathrm{c}}$ \\ ${ }^{a}$ Department of Theoretical Physics, Kazan State Pedagogical University, Mezhlauk Street, \\ 420021 Kazan, 1 Russia \\ ${ }^{\mathrm{b}}$ Department of Physics, University of Augsburg, Universitätsstrasse 1, D-86135 Augsburg, Germany \\ ${ }^{\mathrm{c} D e p a r t m e n t}$ of Psychology, Kazan State Pedagogical University, Mezhlauk Street, \\ 420021 Kazan, 1 Russia
}

Received 20 December 2001

\begin{abstract}
In this paper, the non-Markov statistical processes and long-range memory effects in human sensorimotor coordination are investigated. The theoretical basis of this study is the statistical theory of non-stationary discrete non-Markov processes in complex systems (Phys. Rev. E 62, 6178 (2000)). The human sensorimotor coordination was experimentally studied by means of standard dynamical tapping test on the group of 32 young peoples with tap numbers up to 400 . This test was carried out separately for the right and the left hand according to the degree of domination of each brain hemisphere. The numerical analysis of the experimental results was made with the help of power spectra of the initial time correlation function, the memory functions of low orders and the first three points of the statistical spectrum of non-Markovity parameter. Our observations demonstrate, that with the regard to results of the standard dynamic tapping-test it is possible to divide all examinees into five different dynamic types. We have introduced the conflict coefficient to estimate quantitatively the order-disorder effects underlying life systems. The last one reflects the existence of disbalance between the nervous and the motor human coordination. The suggested classification of the neurophysiological activity represents the dynamic generalization of the well-known neuropsychological types and provides the new approach in a modern neuropsychology.
\end{abstract}

(c) 2002 Elsevier Science B.V. All rights reserved.

PACS: 05.45.-a; 05.45.Tp; 82.40.Bj; 89.75.Fb

Keywords: Complex systems; Non-Markov effects; Human sensorimotor coordination

\footnotetext{
${ }^{*}$ Corresponding author. Tel.: 7-8432-32-07-79; fax: +7-8432-32-42-69.

E-mail address: rmy@dtp.ksu.ras.ru (R.M. Yulmetyev).
} 


\section{Introduction}

From the viewpoint of neurophysiological control, the sensorimotor coordination is important because it signifies human individual differences [1]. The fundamental rules of brain functioning mechanisms responsible for this coordination are unknown and complicated. In particular, it is a well-established fact that features of interhemispheres interactions of human brain are intimately connected to the specificity of fulfillment of different cognitive tasks. It is well known, that the interhemispheres organization of brain activity is one of the major factors which renders appreciable influence on the character and features of realization of mental functions of a person [2]. Recently, Chen et al. [1] discovered that the task of synchronizing the movement of one's limb to a periodic environmental signal can be modeled by the fractional Gaussian noise and the stochastic delayed differential equations.

Long ago it was suggested that many complex natural systems should be described by some of low dimensional non-linear dynamic models. The properties of these systems are expressed by Lyapunov exponents, unique fractal dimensions or Kolmogorov-Sinai entropy. However, considerable recent attention has been focused on the wide variety of complex systems and it revealed convincingly that such low dimensionality could be expected for the coherent phenomena like the ones observed in laser systems. Alive data seems to have a more complicated structure largely due to high-dimensional and many-factor processes and due to the pronounced effects of random fluctuations and long-time memory effects.

Since publications [1], the influence of long-range correlations for human brain activity and sensorimotor coordination has been revealed. In addition to it the analysis presented in [1] demonstrate that the random time series associated with the synchronization task contain long-term memory. As shown in Refs. [3,4], the functional significance of long memory in human coordination may be related to the flexibility of switches from one pattern to another. For the description of the effects of the time correlation in complex systems the standard methods of statistical physics are basically used. Among them is the fractal approach, based on scaling of the frequency spectrum on power law $\omega^{-\alpha}$ [5]. Some properties of such systems are successfully described with the help of the theory of self-organized criticality [6] or Hurst exponent [7] as well as the methods of non-linear dynamics (see Refs. [8-15]).

In alive systems the time fluctuations of independent parameters play an important role [1]. Moreover, the scale and the intensity of time correlations, the long-range memory effects, the discrete and intermittent nature of the experimental procedure of measurement is very important. The radical change of different regimes of behavior and alternation of various types of functioning mechanisms is peculiar to alive systems. Here, we use the statistical theory of discrete non-Markov stochastic processes given in Ref. [16] for the more detail description of the dynamic properties of alive complex systems. It is a well-established fact, that the non-Markov properties and long-range statistical memory effects play a great role in the behavior of complex alive systems $[17,18]$. For this reason, we take into account the full set of statistical non-Markov 
parameters and functions. Further, on we shall use the power spectra of low order memory functions as well as the first three points of non-Markovity parameter for the analysis of the effects of discretization, memory and intermittency of the underlying processes. The detailed technique of the calculations of these quantities was considered in Ref. [16].

The brain activity and, in particular, the laws of hemisphere interaction, are one of the striking examples of complex systems. Recently, the study of these systems has attracted great attention [3-14]. Many of psychological and physiological researches are based on EEGs [13]. The parameter measured in these studies is the potential of group of neurons or an individual neuron of a brain. The changes of this parameter within a short interval of time, about $10^{-3}-10^{-1} \mathrm{~s}$, are usually analyzed. The self-organized criticality is frequently observed in the dynamics of EEGs. It comes from the characteristic fractal behavior of power spectra $P(f) \sim f^{-\alpha}[10]$.

On the other hand, macroscopical parameters should also exist here. The study of long-term memories in human coordination [11] is one of the most available standard psychological tests.

In such investigations the macroscopic parameter, which characterizes the system as a whole at the given moment and depends on full set of the available microscopic parameters, is measured.

The static tests are used traditionally in psychology, and the measured parameter does not depend on time, as a rule. The dynamics of parameter is not usually taken into account. One of central positions of the neuropsychological theory of cerebral organization of the highest mental functions says, that brain works as a paired organ for any mental function. The laws of interaction and asymmetry of hemispheres, taken as the specific case of interaction, can be referred to the fundamental laws of brain activity as a paired organ. The parameters of hemisphere asymmetry correlates with different mental processes. In psychological literature the notion "hemispheresness" is widely use. Most of the authors understand it as "handness", that is preference of the right or left hand or their equality. The connection of "hemispheresness" ("handness") with the emotional-personal sphere and the cognitive processes is well-known. There is also a connection of "handness" with certain features of human temperament. With regard to the domination of brain hemispheres the following classification is developed in psychology [2]: (i) the "plain" right-hander, (ii) the right-hander, (iii) the mixed type or ambidexter, (iv) the left-hander and (v) the "plain" left-hander. Such division is created on the basis of static tests, questionnaires and the self-estimation of examinees.

In the present paper, we investigate long-term memory effects in sensorimotor human's reaction with the help of the statistical theory of discrete non-Markov processes [16]. In our study, we use the experimental results of dynamic tapping-test. The experimental data were obtained by the dynamic tapping-test for the left and the right hand. In Section 2, we briefly discuss the basic points of our statistical theory. In Section 3, the experimental method is shown. In Section 4, we analyze the obtained figures and parameters, and in Section 5 we discuss our results. 


\section{Basic concepts and definitions}

We use the theory of discrete non-Markov processes in complex systems developed in the recent paper [16]. Below we present the basic points of this theory.

Analyzing the complex system we obtain the discrete equidistant series of the experimental data, the so-called random variable

$$
X=\{x(T), x(T+\tau), x(T+2 \tau), \ldots, x(T+k \tau), \ldots, x(T+\tau N-\tau)\} .
$$

It corresponds to the measured signal within time $t=(N-1) \tau$, where $\tau$ is a temporary sampling interval of the signal. The mean value $\langle X\rangle$, fluctuation $\delta x_{j}$, absolute $\left(\sigma^{2}\right)$ and relative $\left(\delta^{2}\right)$ dispersion for the set of random variables (2.1) are defined as follows

$$
\begin{aligned}
& \langle X\rangle=\frac{1}{N} \sum_{j=0}^{N-1} x(T+j \tau), \\
& x_{j}=x(T+j \tau), \quad \delta x_{j}=x_{j}-\langle X\rangle, \\
& \sigma^{2}=\frac{1}{N} \sum_{j=0}^{N-1} \delta x_{j}^{2}, \\
& \delta^{2}=\frac{\sigma^{2}}{\langle X\rangle^{2}}=\frac{1 / N \sum_{j=0}^{N-1} \delta x_{j}^{2}}{\left\{1 / N \sum_{j=0}^{N-1} x(T+j \tau)\right\}^{2}} .
\end{aligned}
$$

The above-mentioned values determine the static (independent from time) property of the considered system. For the dynamical analysis, it is more convenient to use the normalized time correlation function (TCF). For the discrete processes the TCF has the regular form $(t=m \tau, N-1 \geqslant m \geqslant 1)$

$$
a(t)=\frac{1}{(N-m) \sigma^{2}} \sum_{j=0}^{N-1-m} \delta x(T+j \tau) \delta x(T+(j+m \tau)) .
$$

The properties of TCF $a(t)$ are determined by the condition of normalization and attenuation of correlations

$$
\lim _{t \rightarrow 0} a(t)=1, \quad \lim _{t \rightarrow \infty} a(t)=0 .
$$

For real systems the values $x_{j}=x(T+j \tau)$ and $\delta x_{j}=\delta x(T+j \tau)$ represent the experimental data. To account the dynamics of a system we shall define the evolution operator $U\left(T+t_{2}, T+t_{1}\right)$ as follows $\left(t_{2} \geqslant t_{1}\right)$ :

$$
x(t+\tau)=U(t+\tau, t) x(t) .
$$

Then the equation of the motion becomes discrete

$$
\frac{\mathrm{d} x}{\mathrm{~d} t}=\frac{\Delta x(t)}{\Delta t}=\mathrm{i} \hat{L}(t, \tau) x(t), \hat{L}(t, \tau)=(\mathrm{i} \tau)^{-1}[U(t+\tau, t)-1] .
$$


Let us introduce the vector of the initial and final states

$$
\begin{aligned}
\mathbf{A}_{k}^{0}(0)= & \left(\delta x_{0}, \delta x_{1}, \delta x_{2}, \ldots, \delta x_{k-1}\right) \\
= & (\delta x(T), \delta x(T+\tau), \ldots, \delta x(T+(k-1) \tau) . \\
\mathbf{A}_{m+k}^{m}(t)= & \left\{\delta x_{m}, \delta x_{m+1}, \delta x_{m+2}, \ldots, \delta x_{m+k-1}\right\} \\
= & \{\delta x(T+m \tau), \delta x(T+(m+1) \tau), \\
& \delta x(T+(m+2) \tau), \ldots, \delta x(T+(m+k-1) \tau\} .
\end{aligned}
$$

The normalized TCF can be presented as a scalar product of the states vectors $(t=m \tau$ is discrete time):

$$
a(t)=\frac{\left\langle\mathbf{A}_{k}^{0} \cdot \mathbf{A}_{m+k}^{m}\right\rangle}{\left\langle\mathbf{A}_{k}^{0} \cdot \mathbf{A}_{k}^{0}\right\rangle}=\frac{\left\langle\mathbf{A}_{k}^{0}(0) \cdot \mathbf{A}_{m+k}^{m}(t)\right\rangle}{\left\langle\mathbf{A}_{k}^{0}(0)^{2}\right\rangle} .
$$

The initial TCF can be received by projecting of the vector of the final state on the vector of the initial state with the help of the following projection operator:

$$
\Pi \mathbf{A}_{m+k}^{m}(t)=\mathbf{A}_{k}^{0}(0) \frac{\left\langle\mathbf{A}_{k}^{0}(0) \mathbf{A}_{m+k}^{m}(t)\right\rangle}{\left\langle\left|\mathbf{A}_{k}^{0}(0)\right|^{2}\right\rangle}=\mathbf{A}_{k}^{0}(0) a(t) .
$$

The projection operator $\Pi$ possesses the following properties:

$$
\begin{aligned}
& \Pi=\frac{\left|\mathbf{A}_{k}^{0}(0)\right\rangle\left\langle\mathbf{A}_{k}^{0}(0)\right|}{\left\langle\left|\mathbf{A}_{k}^{0}(0)\right|^{2}\right\rangle}, \quad \Pi^{2}=\Pi, \quad P=1-\Pi, \quad P^{2}=P, \\
& \Pi P=0, \quad P \Pi=0 .
\end{aligned}
$$

The vector of the fluctuation obeys the finite-difference Liouville's equation

$$
\frac{\Delta}{\Delta t} \mathbf{A}_{m+k}^{m}(t)=\mathrm{i} \hat{L}(t, \tau) \mathbf{A}_{m+k}^{m}(t) .
$$

The projection operators $\Pi$ and $P$ split the Euclidean space of states $A(k)$ into two mutually-orthogonal subspaces which allows us to split the dynamic equation (2.15) into two equations in two subspaces:

$$
\begin{aligned}
& \frac{\Delta A^{\prime}(t)}{\Delta t}=\mathrm{i} \hat{L}_{11} A^{\prime}(t)+\mathrm{i} \hat{L}_{12} A^{\prime \prime}(t), \\
& \frac{\Delta A^{\prime \prime}(t)}{\Delta t}=\mathrm{i} \hat{L}_{21} A^{\prime}(t)+\mathrm{i} \hat{L}_{22} A^{\prime \prime}(t) .
\end{aligned}
$$

Extracting from Eq. (2.17) the irrelevant part $\Delta A^{\prime \prime}(t)$ we obtain the closed finitedifference equation of a non-Markov type for TCF $a(t)$ :

$$
\frac{\Delta a(t)}{\Delta t}=\lambda_{1} a(t)-\tau \Lambda_{1} \sum_{j=0}^{m-1} M_{1}(j \tau) a(t-j \tau) .
$$


Here, $\Lambda_{1}$ is a relaxation parameter with the dimension of square of frequency and parameter $\lambda_{1}$ describes an eigen-spectrum of Liouville's quasioperator $\hat{L}$

$$
\lambda_{1}=\mathrm{i} \frac{\left\langle\mathbf{A}_{k}^{0}(0) \hat{L} \mathbf{A}_{k}^{0}(0)\right\rangle}{\left\langle\left|\mathbf{A}_{k}^{0}(0)\right|^{2}\right\rangle}, \quad \Lambda_{1}=\frac{\left\langle\mathbf{A}_{k}^{0} \hat{L}_{12} \hat{L}_{21} \mathbf{A}_{k}^{0}(0)\right\rangle}{\left\langle\left|\mathbf{A}_{k}^{0}(0)\right|^{2}\right\rangle} .
$$

The function $M_{1}(j \tau)$ in the r.h.s. of Eq. (2.18) represents the first memory function

$$
M_{1}(j \tau)=\frac{\left\langle\mathbf{A}_{k}^{0}(0) \hat{L}_{12}\left\{1+\mathrm{i} \tau \hat{L}_{22}\right\}^{j} \hat{L}_{21} \mathbf{A}_{k}^{0}(0)\right\rangle}{\left\langle\mathbf{A}_{k}^{0}(0) \hat{L}_{12} \hat{L}_{21} \mathbf{A}_{k}^{0}(0)\right\rangle}, \quad M_{1}(0)=1 .
$$

It is easy to notice, that except for the initial TCF in Eq. (2.20) we consider the time correlation of the new orthogonal dynamic variable $\hat{L}_{21} \mathbf{A}_{k}^{0}(0)$.

Eq. (2.18) represents the first equation of the chain of finite-difference kinetic equations with memory for the discrete TCF $a(t)$. The memory function $M_{1}(t)$ takes into account the memory about the previous states of the system. Acting similarly to the above-stated procedure one can receive kinetic equations for subsequent memory functions. However, a more convenient way is making use of the Gram-Schmidt orthogonalization procedure [16]. Because of this it is easy to obtain the recurrence formula, in which variables $\mathbf{W}_{n}=\mathbf{W}_{n}(t)$ with higher index are expressed in terms of the variables with lower indices

$$
\begin{aligned}
& \mathbf{W}_{0}=\mathbf{A}_{k}^{0}(0), \quad \mathbf{W}_{1}=\left\{\mathrm{i} \hat{L}-\lambda_{1}\right\} \mathbf{W}_{0}, \ldots \\
& \mathbf{W}_{n}=\left\{\mathrm{i} \hat{L}-\lambda_{n-1}\right\} \mathbf{W}_{n-1}+\Lambda_{n-1} \mathbf{W}_{n-2}+\ldots, \quad n>1 .
\end{aligned}
$$

Using the above-mentioned procedure and introducing the corresponding projection operators, we come to the following chain of connected non-Markov finite-difference kinetic equations $(t=m \tau)$

$$
\frac{\Delta M_{n}(t)}{\Delta t}=\lambda_{n+1} M_{n}(t)-\tau \Lambda_{n+1} \sum_{j=0}^{m-1} M_{n+1}(j \tau) M_{n}(t-j \tau) .
$$

Here, parameters $\lambda_{n+1}$ represent an eigen values of the Liouville's quasioperator and the relaxation parameters of $\Lambda_{n+1}$ are determined as follows:

$$
\lambda_{n}=\mathrm{i} \frac{\left\langle\mathbf{W}_{n} \hat{L} \mathbf{W}_{n}\right\rangle}{\left\langle\left|\mathbf{W}_{n}\right|^{2}\right\rangle}, \quad \Lambda_{n}=-\frac{\left\langle\mathbf{W}_{n-1}\left(\mathrm{i} \hat{L}-\lambda_{n+1}\right) \mathbf{W}_{n}\right\rangle}{\left\langle\left|\mathbf{W}_{n-1}\right|^{2}\right\rangle} .
$$

The zero-memory function $M_{0}(t)(2.22)$

$$
M_{0}(t)=a(t)=\frac{\left\langle\mathbf{A}_{k}^{0}(0) \mathbf{A}_{m+k}^{m}(t)\right\rangle}{\left\langle\left|\mathbf{A}_{k}^{0}(0)\right|^{2}\right\rangle}, \quad t=m \tau
$$

describes the statistical memory in complex systems with discrete time. The initial TCF $\mathrm{a}(\mathrm{t})$ and the set of discrete memory functions $M_{n}(t)$ in Eq. (2.22) are important for further consideration. The first three equations of this chain $(t=m \tau$ is discrete time) 
can be presented as follows:

$$
\begin{aligned}
& \frac{\Delta a(t)}{\Delta t}=-\tau \Lambda_{1} \sum_{j=0}^{m-1} M_{1}(j \tau) a(t-j \tau)+\lambda_{1} a(t), \\
& \frac{\Delta M_{1}(t)}{\Delta t}=-\tau \Lambda_{2} \sum_{j=0}^{m-1} M_{2}(j \tau) M_{1}(t-j \tau)+\lambda_{2} M_{1}(t), \\
& \frac{\Delta M_{2}(t)}{\Delta t}=-\tau \Lambda_{3} \sum_{j=0}^{m-1} M_{3}(j \tau) M_{2}(t-j \tau)+\lambda_{3} M_{2}(t) .
\end{aligned}
$$

These systems of finite-difference equations (2.22) and (2.23) are a discrete analogue of the well-known chain of kinetic Zwanzig'-Mori's equations. The latter plays a fundamental role in modern statistical physics of non-equilibrium phenomena with continuous time. It is necessary to note that the chain of Zwanzig'-Mori's equations is valid only for quantum and classical Hamiltonian systems with continuous time. The finite-difference chain of kinetic equations (2.22),(2.23) is valid for complex systems, in which there is no Hamiltonian, time is discrete, and there are no exact equations of motion. However, the "dynamics" and "motion" in real complex systems undoubtedly exist and can be directly registered in the experiment. The first three equations in the chain (2.23) form the basis for quasihydrodynamic description of stochastic discrete processes in complex systems. Below we reproduce as an example the calculation of the first memory function for $m$-discrete steps, assuming that parameters $\lambda_{1}=0, \lambda_{2}=0$, $\lambda_{3}=0$ :

$$
\begin{aligned}
& M_{1}(0)= 1, \quad M_{1}(\tau)=-a(2 \tau)+\epsilon_{1}^{-1}\{a(2 \tau)-a(3 \tau)\} \\
& M_{1}(2 \tau)=-\left\{a(2 \tau) M_{1}(\tau)+a(3 \tau)\right\}+\epsilon_{1}^{-1}\{a(3 \tau)-a(4 \tau)\}, \\
& M_{1}(3 \tau)=-\left\{a(2 \tau) M_{1}(2 \tau)+a(3 \tau) M_{1}(\tau)+a(4 \tau) M_{1}(0)\right\}+\epsilon_{1}^{-1}\{a(4 \tau)-a(5 \tau)\}, \\
& \vdots \\
& M_{1}(m \tau)=-\sum_{j=0}^{m-1} M_{1}(j \tau) a\{(m+1-j) \tau\}+\epsilon_{1}^{-1}[a\{(m+1) \tau\} \\
&\quad-a\{(m+2) \tau\}] .
\end{aligned}
$$

Generally, we can find the recurrent relations between memory functions of higher and lower orders which are

$$
\begin{aligned}
& M_{s}(m \tau)=- \sum_{j=0}^{m-1} M_{s}(j \tau) M_{s-1}((m+1-j) \tau) \\
&+ \epsilon_{s}^{-1}\left\{M_{s-1}((m+1) \tau)-M_{s-1}((m+2) \tau)\right\}, \\
& \epsilon_{s}=\tau^{2} \Omega_{s}^{2}, \quad s=1,2,3, \ldots
\end{aligned}
$$


The obtained relations allow us to find the necessary memory function $M_{s}(t)$ of any order $s=1,2 \ldots$ on the basis of the experimental data, using only the initial TCF $a(m \tau)$ [17]. Relaxation parameters $\lambda_{i}$ and $\Lambda_{i}, i=1,2,3 \ldots$, in Eqs. (2.24) are evaluated from the experimental data. The application of Eqs. (2.25) opens new possibilities in the detailed analysis of the statistical properties of the correlations in complex systems. The fact of the existence of a finite- difference Eqs. (2.22) allows us to evaluate unknown functions directly from the experimental data.

\section{Technique of experiment and experimental data processing}

Thirty two students of the physical faculty of the pedagogical university aged from 19 to 22 took part in this research. The research included 400 approaches of the tapping-test for the right hand for $5 \mathrm{~s}$. The obtained data were processed with the help of the above introduced technique. The set of three memory functions was calculated for each sequence of data. Frequency power spectra for each of these functions are obtained with the help of the fast Fourier transform. For a more detailed diagnostics of properties of the system we also consider the frequency spectrum of the first three points of the statistical spectrum of non-Markovity parameter. The spectrum of non-Markovity parameter was entered earlier in Refs. [19,20] and was also used in statistical physics of liquids [4]. In this study, we use frequency spectrum of non-Markovity parameter

$$
\varepsilon_{i}(\omega)=\left\{\frac{\mu_{i-1}(\omega)}{\mu_{i}(\omega)}\right\}^{1 / 2} .
$$

Here, $i=1,2$.. and $\mu_{i}(\omega)$ there is a power spectrum of $i$ th level. The parameters $\varepsilon_{i}$ allow to receive quantitative estimation of long-term memory effects in experimental time series of the data as shown in apers [4,19-21]. From the physical point of view parameter $\varepsilon_{i}$ allows to mark out the three most important cases [4,19-21]. The Markov and completely random processes correspond to values $\varepsilon \rightarrow \infty$, quasi-Markov processes (elements of memory can be noticed there) correspond to values $\varepsilon>1$. The limiting case $\varepsilon \sim 1$ concerns the case of non-Markov processes, i.e., processes, where there is a long-term memory.

All the examinees were distributed into five representative groups by analogy to the neuropsychological classification [2] and by the obtained spectra of memory functions and the first points of non-Markovity parameter. Among them: (i) the "plain" right-hander, (ii) the right-hander, (iii) the mixed type or ambidexter, (iv) the lefthander and (v) the "plain" left- hander.

\section{Discussion}

In this section, in Figs. 1-7, we present the characteristic behavior of memory functions, their power spectra and frequency spectra of the first three points of nonMarkovity parameter for each representative group. 

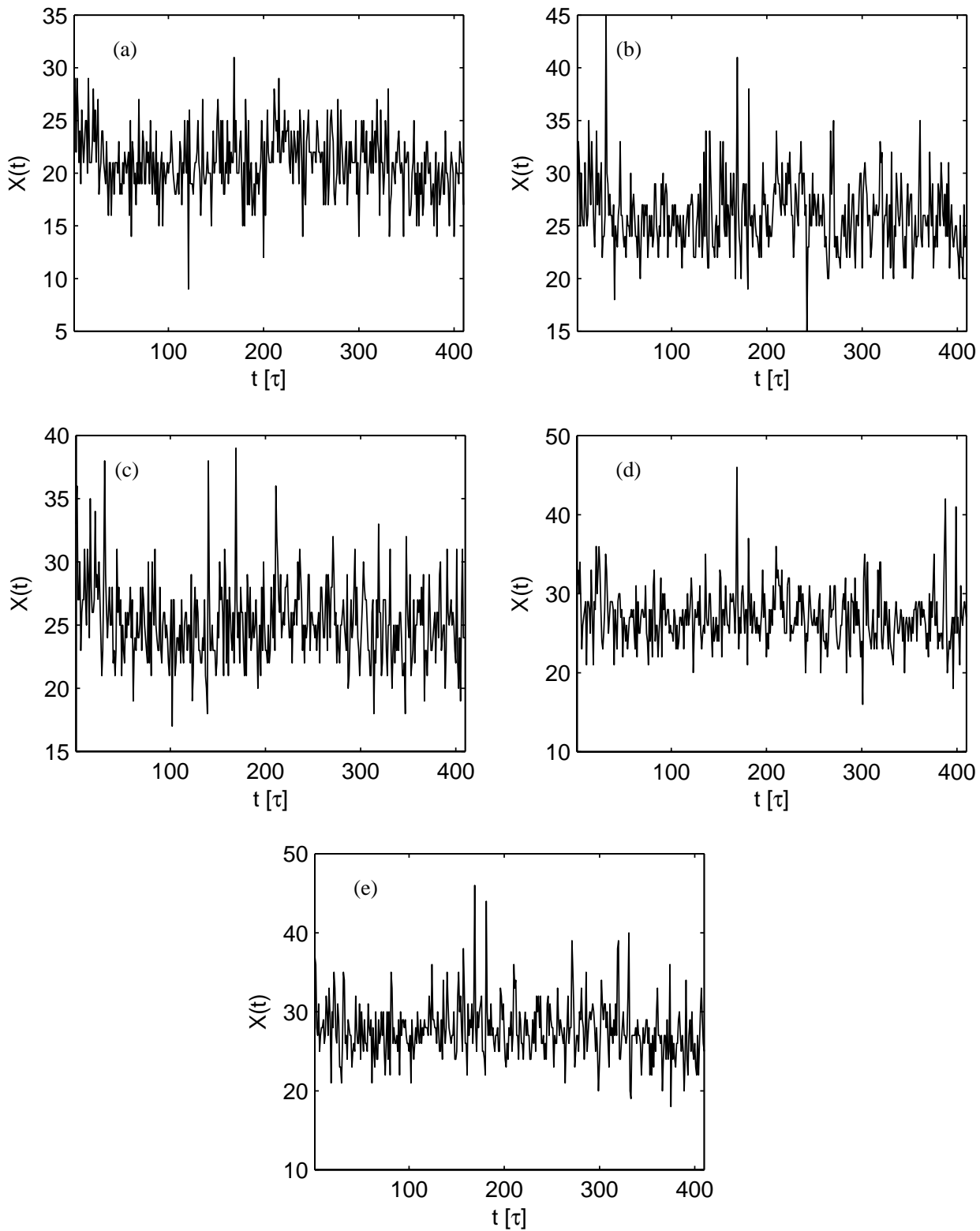

Fig. 1. Time recording of measured taping-test parameter for five types of examinees: (a) I type ("plain" right-hander), (b) II type (right-hander), (c) III type (ambidexter or mixer type), (d) IV type (left-hander), (e) V type ("plain" left-hander). 

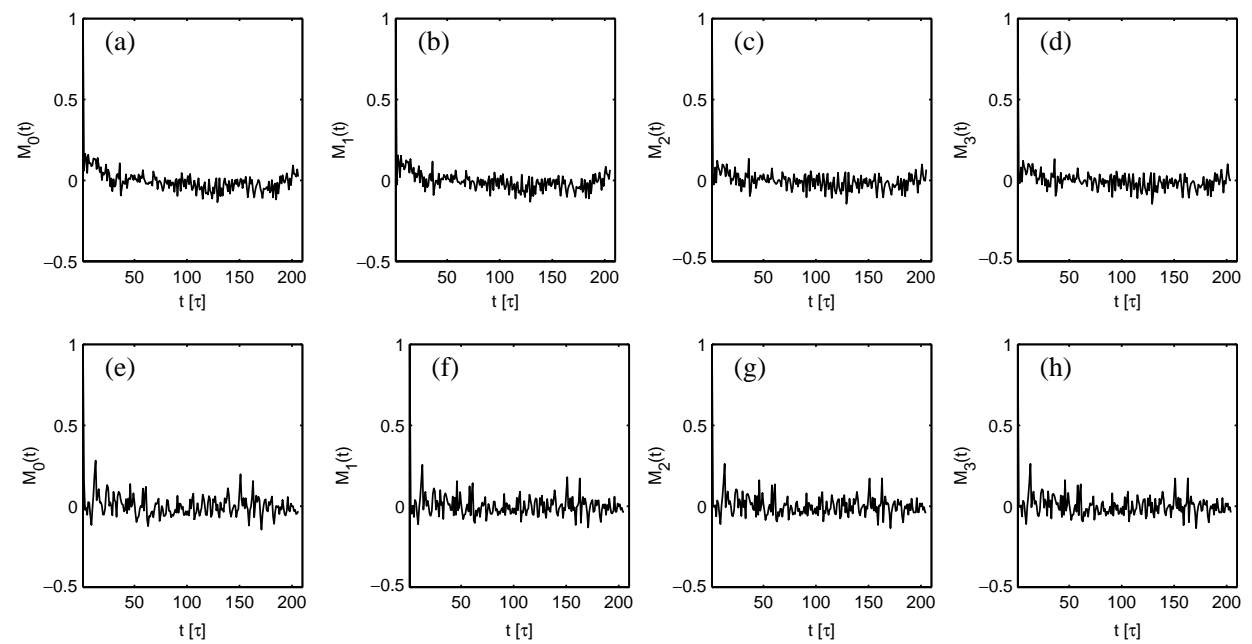

Fig. 2. Time dependence of initial time correlation function $M_{0}(t)$ : (a, e); first-order memory function $M_{1}(t)$ : $(\mathrm{b}, \mathrm{f})$; second-order memory function $M_{2}(t)$ : $(\mathrm{c}, \mathrm{g})$ and third-order memory function $M_{3}(t)$ : $(\mathrm{d}, \mathrm{h})$ for type I (a-d) and for type V (e-h).

In Fig. 1, the time recording of the initial dynamic variable $X(t)$ of the dynamic tapping-test is given for all the characteristic groups. Both weak and strong fluctuations of the measured parameter are visible in Figs. 1a-e. The greatest fluctuations are appreciable in case 1e ("clean" left-hander) and 1d (left-hander). The least fluctuations are appreciable in case 1a ("clean" right-hander) and 1c (ambidexter or mixed type). In Fig. 2, the time dependence of TCF and three memory functions are given for the two extreme cases: type I Figs. 2a-d) and type V (Figs. 2e-h). From these figures it is visible, that high-frequency modulation is characteristic of type I. On the other hand, intensive and sharp correlations in the dynamics for type $\mathrm{V}$ are appreciable for the parameter of the tapping-test. In Figs. 3-5, the power spectra for the initial TCF and the first three memory functions for types I, III and V are given. The fractal behavior, which is characterized by a sharp growth of intensity on low frequencies, is visible in Fig. 3 for all four spectra $(a-d)$ of type I. Such behavior is characteristic for the steadiest type of human sensorimotor coordination. Thus, appreciable peaks on high frequencies occur only in senior memory functions (see Figs. 3c and d). This clearly demonstrates the harmonious, synchronized and coordinated activity of human motor and nervous subsystems. The similar behavior varies gradually at transition from type I to type III and then and to type V (see Figs. 4 and 5). Peaks increase everywhere for type III, and noticeable peaks arise in all spectra for type V. Moreover, the height of zero peaks in case of type $\mathrm{V}$ decreases almost 6 times. The experimental data testify that in systems III and V the mismatch between motor and nervous subsystems arises and appreciably amplifies. Between them there is a conflict, which can be described quantitatively.

The frequency spectra of the first three points of non-Markovity parameter, introduced in Figs. 6a-i are of particular interest. The long-range memory is easily found 

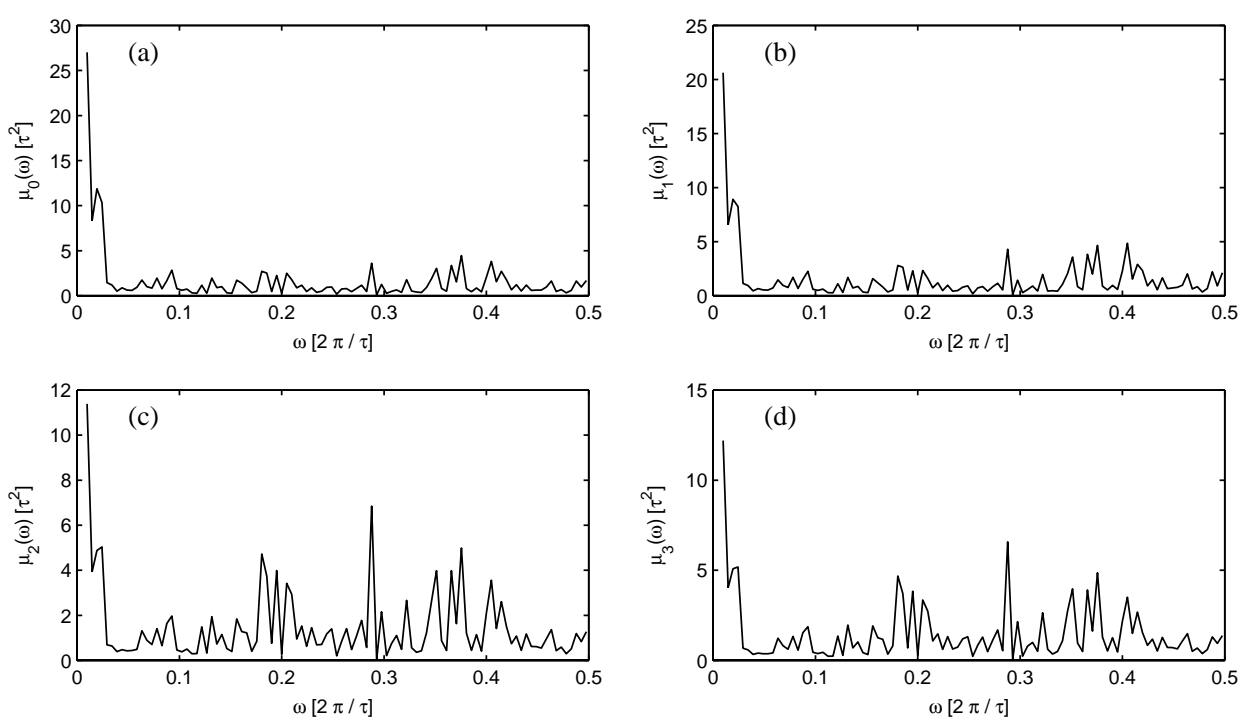

Fig. 3. Power spectra of initial time correlation function $\mu_{0}(\omega)$ : (a), of first-order $\mu_{1}(\omega)$ : (b), second-order $\mu_{2}(\omega)$ : (c) and third-order $\mu_{3}(\omega)$ : (d) memory functions for type I of examinees.
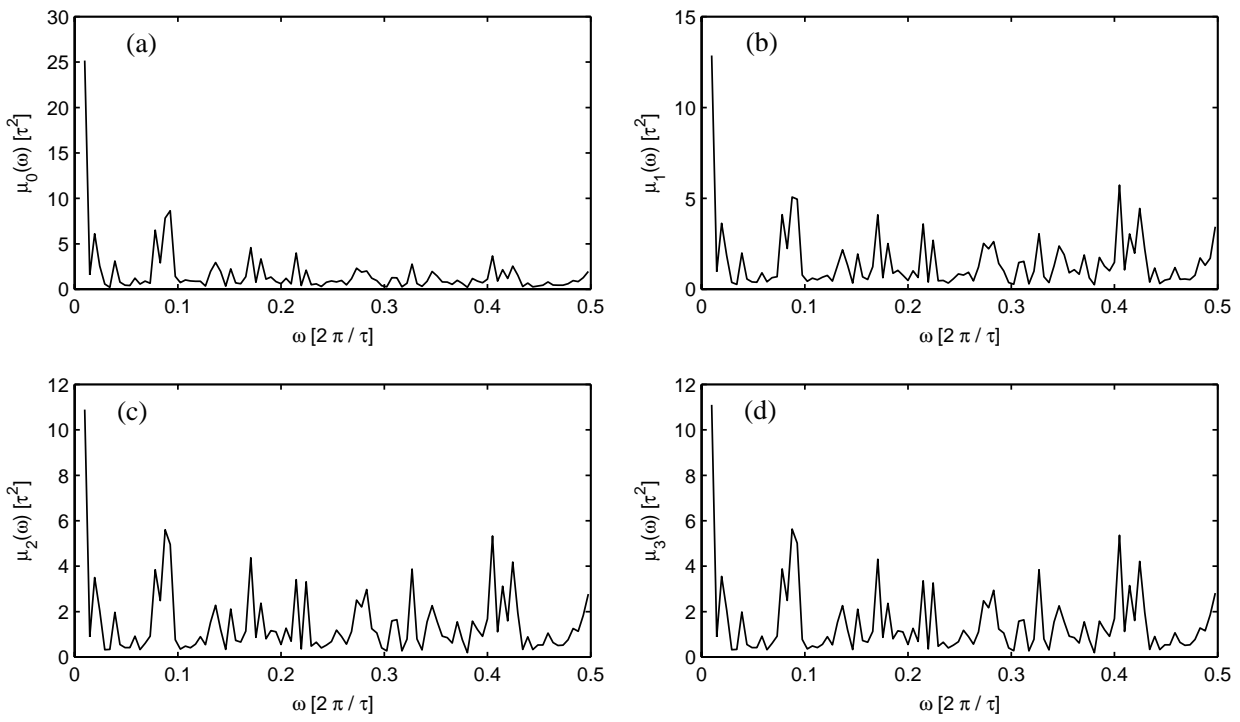

Fig. 4. Power spectra of initial time correlation function $\mu_{0}(\omega)$ : (a) and first-order $\mu_{1}(\omega)$ : (b), second-order $\mu_{2}(\omega)$ : (c) and third-order $\mu_{3}(\omega)$ : (d) memory functions for type III of examinees. 

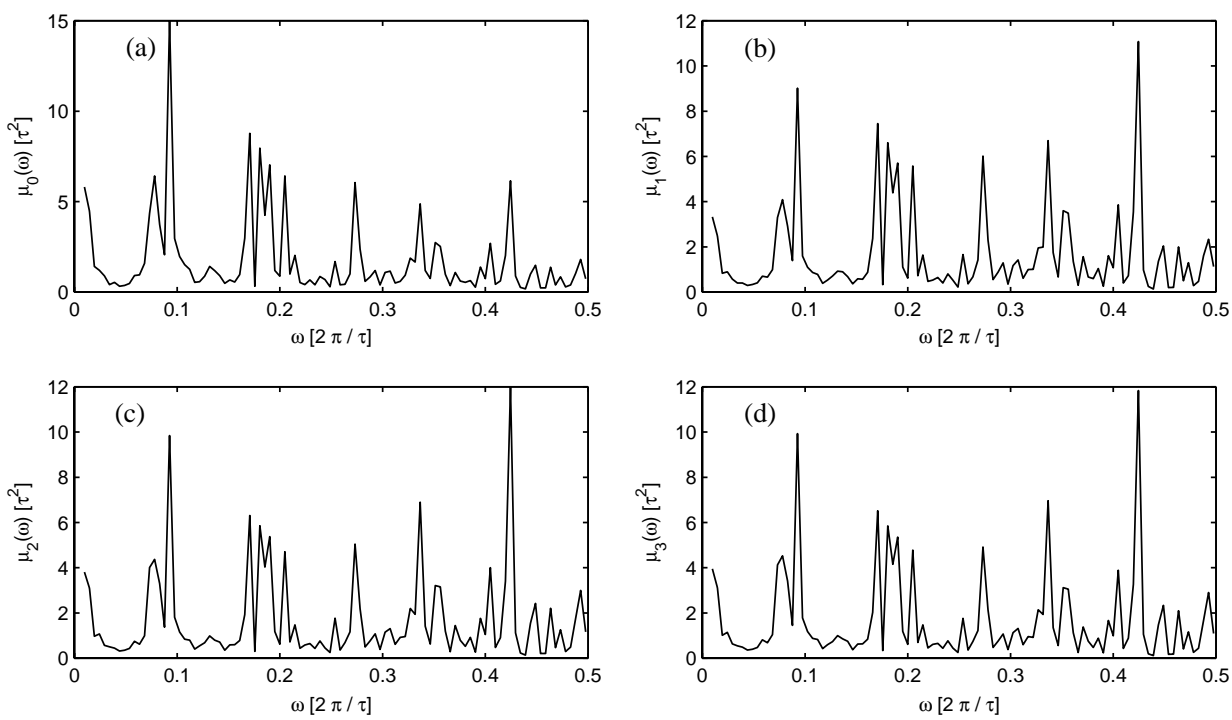

Fig. 5. Power spectra of initial time correlation function $\mu_{0}(\omega)$ : (a) and first-order $\mu_{1}(\omega)$ : (b), second-order $\mu_{2}(\omega)$ : (c) and third-order $\mu_{3}(\omega)$ : (d) memory functions for type $\mathrm{V}$ of examinees.
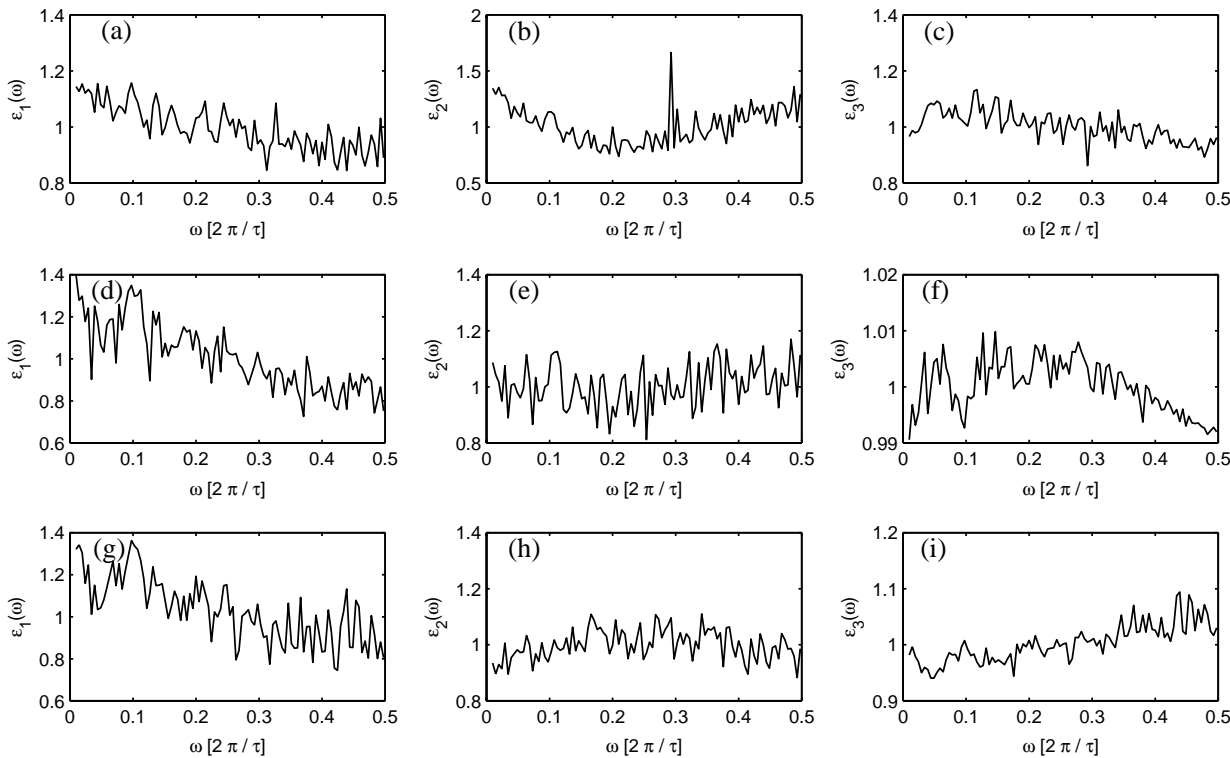

Fig. 6. Frequency spectra of the first three points of non-Markovity parameter $\epsilon_{1}(\omega):(a, d, g)$, $\epsilon_{2}(\omega):(b, e, h), \epsilon_{3}(\omega):(c, f, i)$ for three most characteristic types of examinees : type I (a-c), type III (d-f), type $\mathrm{V}(\mathrm{g}-\mathrm{i})$. 

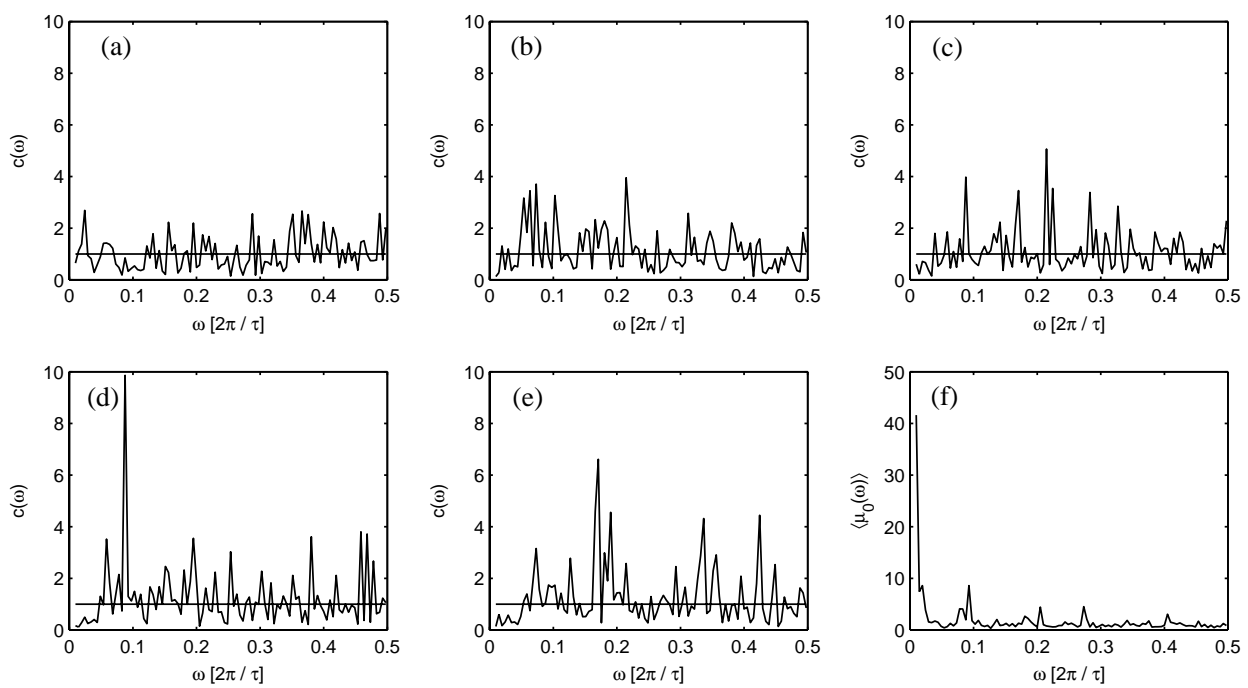

Fig. 7. Frequency dependence of a conflictness coefficient $\mathrm{c}(\omega)$ for the typical representatives of five types of examinees: (a) for type I, (b) for type II, (c) for type III, (d) for type IV and (e) for type V, (f) a mean power spectrum of initial time correlation function $\left\langle\mu_{0}(\omega)\right\rangle$ for the whole of group of examinees of type I.

on frequency dependences of values of parameters $\epsilon_{i}(\omega), i=1,2,3$ for all types $\mathrm{I}-\mathrm{V}$. The presence of rigid memories at the first relaxation level (see, Figs. 6a, d, g) is especially striking. In Figs. 7a-e the frequency spectrum of conflict coefficient $c(\omega)$ is shown for all types of the test. It was evaluated as follows. The mean power spectrum of the initial TCF

$$
\mu_{0}^{(i)}(\omega)=\frac{1}{n_{i}} \sum_{j=1}^{n_{i}} \mu_{0}^{(i, j)}(\omega)
$$

was calculated for all types of the examinees. Here, $\mu_{0}^{(i, j)}(\omega)$ is a power spectrum of $j$ th examinees in $i$ th group, and $n_{i}$ stands for the number of such examinees. The mean spectrum for group I is shown in Fig. 7f. Conflict coefficient for values $i=1,2,3,4,5$ was calculated for the typical representatives of each group as follows:

$$
c^{(i)}(\omega)=\frac{\mu_{0}^{(i)}(\omega)}{\mu_{0}^{(I)}(\omega)} .
$$

The following features are of importance. The conflict coefficient for a representative of group I is close to unit everywhere (see, Fig. 7a). The amplitude of fluctuations $c(\omega)$ increases noticeably in the process of rising conflict between human motor and nervous subsystems. The fluctuations in groups IV and V are especially great. Here, there is a strong mismatch in human sensorimotor activity.

Besides, it is possible to draw the following conclusions of common nature. Great differences between different types of the examinees for various spectra are visible from the given figures. The stably functioning system is in the state of self-organized 
Table 1

Sets of static and kinetic relaxation parameters for typical representatives of five neurophysiological groups

\begin{tabular}{|c|c|c|c|c|c|}
\hline Parameters & I type & II type & III type & IV type & $\mathrm{V}$ type \\
\hline$\langle n\rangle$ & 20.9659 & 26.0244 & 25.2634 & 27.039 & 27.6537 \\
\hline$\sigma^{2}$ & 9.3631 & 11.6375 & 10.0576 & 12.2576 & 12.7355 \\
\hline$\delta$ & 0.14595 & 0.13108 & 0.12553 & 0.12948 & 0.12905 \\
\hline$\lambda_{1},\left[\tau^{-1}\right]$ & -0.93572 & -0.79773 & -0.83812 & -0.80362 & -0.84341 \\
\hline$\lambda_{2},\left[\tau^{-1}\right]$ & -1.0225 & -1.0012 & -1.0243 & -1.0066 & -1.0053 \\
\hline$\lambda_{3},\left[\tau^{-1}\right]$ & -1.0198 & -1.0064 & -1.0011 & -1.0013 & -0.9994 \\
\hline$\Lambda_{1},\left[\tau^{-2}\right]$ & -0.1655 & -0.0216 & -0.0343 & -0.0114 & 0.0445 \\
\hline$\Lambda_{2},\left[\tau^{-2}\right]$ & 0.00689 & 0.00249 & 0.00485 & -0.00825 & -0.00086 \\
\hline$\epsilon_{1}(0)$ & 1.1444 & 1.3307 & 1.3985 & 1.4013 & 1.3211 \\
\hline$\epsilon_{2}(0)$ & 1.3461 & 1.1041 & 1.0866 & 1.0467 & 0.93511 \\
\hline$\epsilon_{3}(0)$ & 0.96614 & 1.0371 & 0.99058 & 1.0137 & 0.98168 \\
\hline
\end{tabular}

criticality, then the spectra of a zero-order memory function for such systems have fractal behavior $1 / \omega^{\alpha}[6]$. For example, power spectrum of TCF for type I (see, Fig. 3a) reminds of the fractal behavior of the systems. In our case it corresponds to domination of the left-hand brain hemisphere (type I). For the examinees of type III power spectrum of TCF is shown in Fig. 4a, it is close to fractal behavior. For the examinees, referred to type $\mathrm{V}$, the power spectrum of TCF looks like a color noise on all frequencies (Fig. 5a). Power spectra of the first, the second and the third memory functions for each type test almost repeat each other with minor characteristic differences. Type I spectra have almost similar behavior in the form of a color noise on the mean and high frequencies (see, Fig. 3b-d). At this point, we can judge about the stability of such a state, and speak about the distinct predominance of the left-hand hemisphere. The frequency spectrum of memory functions of junior orders looks almost like a white noise with a sharp flash of power in a high-frequency region (Figs. $4 b-d)$. It speaks about a stable state of the system, and we refer such examinees to mixed type III.

The complex pattern of spikes is also visible in the spectra of non-Markovity parameter $\varepsilon_{1}(\omega), \varepsilon_{2}(\omega), \varepsilon_{2}(\omega)$ (Fig. 6a-i). These figures demonstrate, that almost all values of non-Markovity parameter $\varepsilon_{1}(\omega), \varepsilon_{2}(\omega) \varepsilon_{3}(\omega)$ lie within a small interval of values (0.6-1.6). This observation strongly suggests that the effects of a long-term memory are exhibited in the dynamics of human sensorimotor coordination.

In Table 1 numerical values of the static $\left(\langle n\rangle, \sigma^{2}, \delta\right)$ and kinetic $\left(\lambda_{1}, \lambda_{2}, \lambda_{3} ; \Lambda_{1}, \Lambda_{2}, \Lambda_{3}\right.$; $\left.\epsilon_{1}(0), \epsilon_{2}(0), \epsilon_{3}(0)\right)$ parameters for the typical representatives of the five neurophysiological groups are given. The average value of the tapping-test parameter $\langle n\rangle$ grows monotonically from the first to the fifth type, and relative dispersion $\delta$ on the contrary decreases. All values of relaxation parameter $\lambda_{i}$ are negative. Let us remind, that in physics, parameter $\lambda_{i}$ is close to Lyapunov's exponent [22]. Behavior $\lambda_{i}$ testifies the stability of the system from the point of view non-linear dynamics $\left(\lambda_{i} \leqslant 0\right)$. The numerical values of another relaxation parameter $\Lambda_{i}$ appears small and sign-variable. 
The numerical values of non-Markovity parameters $\epsilon_{i}(0), i=1,2,3$ are within an interval $0.966-1.401$, that manifests the rigid prevalence of the long-term memory in the dynamics of parameter of the tapping-test.

\section{Conclusion}

In this paper, we have obtained some relevant results. We consider the dynamics of neurophysiological parameter of the tapping-test to be a discrete random non-Markov process. It has allowed us to use the results of the last theory of stochastic non-Markov processes in complex systems [16]. The theory [16] allows to find the functions and parameters of a long-term memory from the experimental data. Our motivation is to present the unifying mechanism for a diverse set of long memory processes, observed under a variety of sensorimotor conditions. On the basis of our experimental data we have calculated memory functions, their power spectra and frequency dependence of non-Markovity parameters.

Our calculus has allowed to find out the dynamic types of neurophysiological activity on the basis of long-term memory data in alive systems. Our classification essentially extends the conventional classification, established in psychology [2]. Besides, we managed to enter new psychological parameter: conflict coefficient. The latter characterizes quantitatively and qualitatively a mismatch and a conflict between the two human neurophysiological subsystems: motor and nervous. The existence of this coefficient enables to describe quantitatively the disbalance in human mental functions.

It is possible to expect, that the given method can be used for the study in other life sciences on the basis of non-Markov properties of statistical memory for these type of complex systems.

We have found a large variety of interesting physical effects in the obtained different non-linear spectra. Among them we have: the fractal-like behavior of the power spectra with power frequency law, the phenomena of self-organized criticality and the restricted self-organized criticality, the spectral behavior of some frequency spectra in the form of white and color noises, the existence of the legibly expressed quality and the quantitative differences in spectral and kinetic characteristics. Our preliminary study shows that the indicated differences can serve as a reliable method in neuropsychological studies.

\section{Acknowledgements}

This work has in part (P.H. and R.Y.) been supported by the Graduiertenkolleg 283: Nonlinear Problems in Analysis, Geometry and Physics, of the Deutsche Forschungsgemeinshaft (DFG), RHSF (Grant (C) 00-06-00005a) and RFBR (Grant (C) 02-02-16146). The authors acknowledge Dr. L.O. Svirina for technical assistance. 


\section{References}

[1] Y. Chen, M. Ding, J.A.S. Kelso, Phys. Rev. Lett. 79 (1997) 4501; J.A.S. Kelso, J.J. Buchanan, G.C. De Guzman, M. Ding, Phys. Lett. A 179 (1993) 364; J.J. Jeka, J.A.S. Kelso, T. Kiemel, Bull. Math. Biol. 55 (1993) 829; P.G. Zanone, J.A.S. Kelso, J. Exp. Psych.: Hum. Percep. Perf. 18 (1992) 403;

B. Tuller, P. Case, M. Ding, J.A.S. Kelso, J. Exp. Psych.: Hum. Percep., Perf. 20 (1994) 3;

G. Schoner, H. Haken, J.A.S. Kelso, Biol. Cybernet. 53 (1986) 247.

[2] E.D. Homskaya, I.V. Efimova, Neuropsychology of Individual Differences, Russian Pedagogical Agency, Moscow, 1997.

[3] L. Ingber, Math. Comput. Modelling 27 (1998) 33; L. Ingber, Phys. Rev. E 55 (1997) 4578.

[4] R.M. Yulmetyev, V.Yu. Shurygin, T.R. Yulmetyev, Physica A 242 (1997) 509; R.M. Yulmetyev, V.Yu. Shurygin, T.R. Yulmetyev, Physica A 212 (1994) 26; R.M. Yulmetyev, V.Yu. Shurygin, N.R. Khusnutdinov, Acta Phys. Pol. B 30 (1999) 881

[5] P. Dutta, P.M. Morn, Rev. Mod. Phys. 53 (1981) 497; M.B. Weissman, Rev. Mod. Phys. 60 (1998) 537.

[6] S. Thurner, S.B. Lowen, M.C. Feurstein, C. Heneghan, Fractals 5 (1997) 565.

[7] J. Davidsen, H.G. Schuster, Phys. Rev. E 62 (2000) 6111.

[8] D. Gribkov, V. Gribkova, Phys. Rev. E 61 (2000) 6538.

[9] W. Duch, Comp. Phys. Comm. 97 (1996) 136; W. Duch, G.H.F. Diercksen, Comp. Phys. Comm. 87 (1995) 341; W. Duch, SAMS 24 (1996) 53.

[10] E. Novikov, A. Novikov, D. Shannahoff-Khalsa, B. Schwartz, J. Wright, Phys. Rev. E 56 (1997) R2387.

[11] J. Kwapien, S. Drozd, L.C. Liu, A.A. Ioannides, preprint.

[12] J. Beran, Statistics for Long-Memory Processes, Chapman \& Hall, New York, 1994.; G. Samorodnitsky, M.S. Taqqu, Stable and Non-Gaussian Random Processes, Chapman \& Hall, New York, 1994.

[13] P.A. Robinson, C.S. Rennie, S.S. Wright, H. Bahramali, E. Gordon, D.L. Rowe, Phys. Rev. E 63 (2001) 021903;

C.J. Rennie, J.J. Wricht, P.A. Robinson, J. Theoret. Biol. 205 (2000) 17;

P.A. Robinson, C.J. Rennie, J.J. Wright, Phys. Rev. E 56 (1997) 826;

P.A. Robinson, J.J. Wright, C.J. Rennie, Phys. Rev. E 57 (1998) 4578.

[14] J.M. Hausdorff, S.L. Mitchell, R. Firtion, C.K. Peng, M.E. Cudkowicz, J.Y. Wel, A.L. Goldberger, J. Appl. Physiol. 82 (1997) 262;

J.M. Hausdorff, P.L. Purdon, C.-K. Peng, Z. Ladin, J.Y. Wel, A.L. Goldberger, J. Appl. Physiol. 80 (1996) 1448;

J.M. Hausdorff, H. Edelberg, L. Michell, A.L. Goldberger, J.Y. Wel, Arch. Phys. Med. Rehabil. 78 (1997) 278.

[15] C.L. Webber, Jr., J.P. Zbilut, J. Appl. Physiol. 76 (1994) 965;

C. Webber, J.P. Zbilut, Recurrent structuring of dynamical and spatial systems, in: A. Colosimo, A. Lesk (Eds.), Complexity in the Living, A Modelistic Approach, Interdisciplinary Science Review, Oxford University Press, New York, 1997.

[16] R. Yulmetyev, P. Hänggi, F. Gafarov, Phys. Rev. E 62 (2000) 6178.

[17] R. Yulmetyev, F.M. Gafarov, Physica A 273 (1999) 416; R. Yulmetyev, F.M. Gafarov, Physica A 274 (1999) 381.

[18] R. Yulmetyev, D.G. Yulmetyeva, Acta Phys. Pol. B 30 (1999) 2511.

[19] V.Yu. Shurygin, R.M. Yulmetyev, V.V. Vorobjev, Phys. Lett. A 148 (1990) 199; V.Yu. Shurygin, R. Yulmetyev, Phys. Lett. A 174 (1993) 433; R. Yulmetyev, R.I. Galeev, V.Yu. Shurygin, Phys. Lett. A 202 (1995) 258. 
[20] V.Yu. Shurygin, R.M. Yulmetyev, Zh. Eksp. Teor. Fiz. 99 (1991) 144;

V.Yu. Shurygin, R.M. Yulmetyev, Zh. Eksp. Teor. Fiz. 102 (1992) 852.

[21] R. Yulmetyev, N.R. Khusnutdinov, J. Phys. A 27 (1994) 5363.

[22] P. Cvitanović, R. Arturo, F. Christiansen, P. Dahlqvist, R. Mainieri, H.H. Rugh, G. Tanner, G. Vattay, N. Whelan, A. Wirzba, Classical and Quantum Chaos, 2000 (www.nbi.dk/ChaosBook version 7.0.1). 